\title{
Decomposition of Core-Loss EEL Edges into a Linear Combination of Refined 'Pure' Spectra
}

\author{
Jacob T. Held ${ }^{1 *}$, Katharine Hunter ${ }^{2}$, Uwe R. Kortshagen ${ }^{2}$ and K. Andre Mkhoyan ${ }^{1}$
}

${ }^{1}$. Department of Chemical Engineering \& Materials Science

2. Department of Mechanical Engineering. University of Minnesota, Minneapolis, MN, USA.

*Corresponding author: heldx123@umn.edu

Core-loss electron energy loss spectroscopy (EELS) is a powerful tool for characterizing elemental composition and electronic structure. When paired with scanning transmission electron microscopy (STEM), two-dimensional spectrum images of EEL spectra can be produced at atomic resolution, enabling the detection of single dopant atoms [1] and local electronic structure [2]. However, only under special circumstances are the EEL edges collected from a single point of a spectrum image representative of a pure material due to plural-scattering, substrates, oxidation, and variation in sample structure.

Even in the case of a thin specimen where single-inelastic-scattering can be assumed, each pixel of an EEL spectrum image is composed of a linear combination of spectra from each atom encountered by the beam in that region [3]. These spectra can be decomposed into a set of 'pure' spectra representative of each class of atom in that region (e.g. crystalline, oxide, and amorphous). In this study, we present a method for decomposing spectra in spectrum images into multiple standards, and for refining these standards. Our approach is similar to established multivariate analysis [4] with the added advantage of producing a set of standards which can then be used as the basis set for further analysis of similar samples. Plasma-synthesized Si nanocrystals (NCs) [5] were used as a test case for demonstration of this technique because they are thin ( $\mathrm{NC}$ diameter $\sim 8 \mathrm{~nm}$ ) and have a simple composition. The NCs were directly deposited onto quantifoil grids and spectrum images of individual NCs were obtained in an FEI-Titan G2 60-300 equipped with a Gatan Enfinium ER EELS spectrometer. Initial 'pure' spectra for amorphous, crystalline, and oxidized Si were obtained under similar conditions from amorphous $\mathrm{Si}$ and a thin FIB lamella of a thermally-oxidized Si wafer, respectively. All spectra were background-subtracted.

Following the flow chart in Figure 1, a linear combination of the reference spectra was fitted to and refined by the Si spectra in each pixel of the spectrum image shown in Figure 2. Over the course of 50 iterations, this procedure produced a best-fit linear combination of the constituent spectra shown in Fig. 2b. This produced maps of the amorphous, crystalline, and oxidized Si in the spectrum image, using only the structure of the Si spectrum. Additional reference spectra, such as $\mathrm{SiC}$, could be added in much the same way to further refine this fitting.

This method yields a linear decomposition of every pixel into refined constituent spectra, which maps the composition and structure (e.g. crystalline vs. amorphous) and can be used to understand the electronic structure of each component. Additionally, the refined standards produced via this technique can be used to fit and remove the Si features from doped NCs of similar size, enabling the detection of heavilyoverlapping dopant signals such as B and P [6].

References:

[1] AA Gunawan, et al., Nano Lett. 11 (2011), p. 5553. 
[2] DA Muller et al., Nature, 399 (1999), p. 758.

[3] RF Edgerton in "Electron Energy-Loss Spectroscopy in the Electron Microscope", (Springer).

[4] F de la Pena et al., Ultramicroscopy 111 (2011), p. 169.

[5] L Mangolini et al., Nano Lett. 5 (2005), p. 655.

[6] This work was supported primarily by NSF through the University of Minnesota MRSEC under Award Number DMR-1420013.

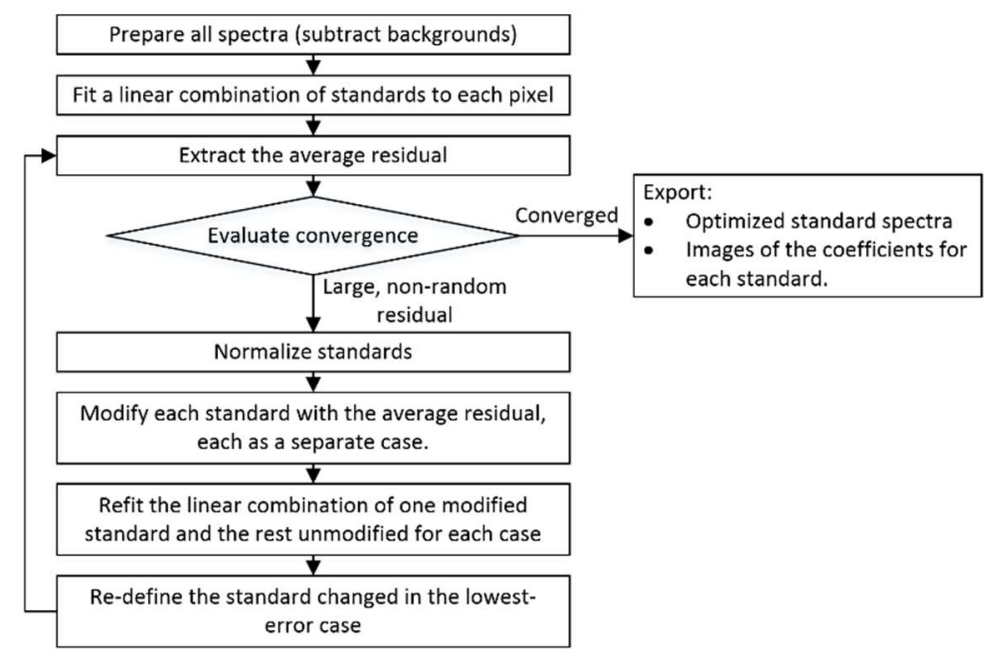

Figure 1. Abbreviated flow chart of the EEL spectrum decomposition and refinement procedure. The background-subtracted spectrum image and reference spectra are input at the start and the loop is repeated until the fitting residual is adequately small.

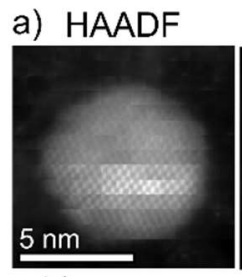

b)

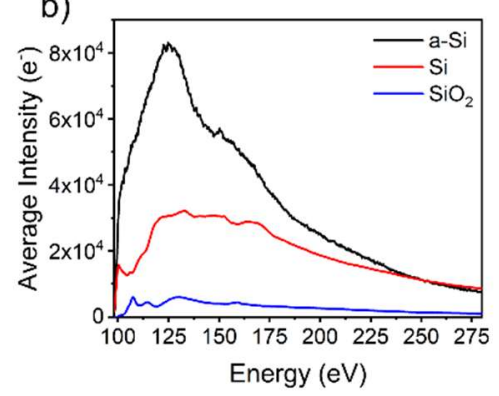

$\mathrm{a}-\mathrm{Si}$

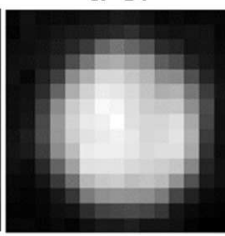

c)

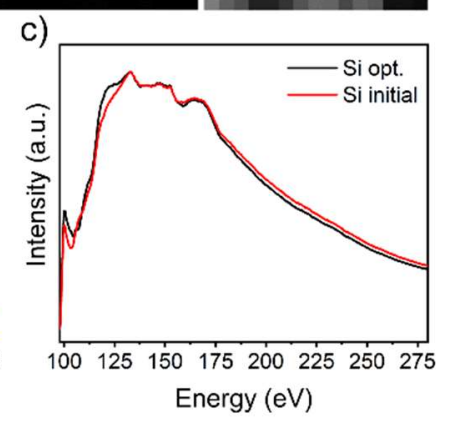

Figure 2. Core-loss decomposition procedure carried out on a spectrum image of a Si NC using externallyprepared standards from pure amorphous $\mathrm{Si}(\mathrm{a}-\mathrm{Si})$, crystalline $\mathrm{Si}(\mathrm{Si})$, and amorphous $\mathrm{SiO}_{2}$ as initial standard spectra. (a) HAADF-STEM image of the Si NC and the coefficients of amorphous, crystalline, and oxide spectra, all rescaled for visibility. Note the apparent core-shell structure of the NC (crystalline $\mathrm{Si}$ core and amorphous shell) as well as the background of $\mathrm{SiO}_{2}$ distributed across the carbon grid. (b) Optimized average constituent spectra for this spectrum image. (c) Comparison of the initial crystalline Si reference vs the refined Si signal. 